\title{
Edge interpretation of potential field data with the normalized enhanced analytic signal
}

\author{
Yongming Yao ${ }^{1,2} \cdot$ Danian Huang ${ }^{1} \cdot$ Xianli $\mathbf{Y u}^{1} \cdot$ \\ Bosen Chai ${ }^{2}$
}

Received: 1 December 2014/ Accepted: 4 May 2015/Published online: 30 May 2015

(C) Akadémiai Kiadó 2015

\begin{abstract}
Edge detection is a requisite task in the interpretation of potential field data. The existed edge detectors have the disadvantages that they cannot balance the edges of strong and weak amplitude anomalies simultaneously, or the identified edges of deep geological bodies are divergence and fuzzy. In order to overcome this problem, this paper presents the normalized enhanced analytic signal detectors to extract the edges, which can improve the disadvantages effectively. The normalized enhanced analytic signal detectors use different orders vertical derivatives of potential field data to normalize the different orders enhanced analytic signals. The new detectors are tested on synthetic gravity data, which can not only display the edges of shallow and deep geological bodies clearly at the same time, also can identify the edges more precisely and clearly. Finally, these detectors are demonstrated on real gravity and magnetic data from China, which can bring out more edge information.
\end{abstract}

Keywords Edge detection · Potential field · Normalized enhanced analytic signal

\section{Introduction}

Edge detection plays an important role in the interpretation of potential-field data, which has been widely used as a tool in exploration technologies for mineral resources. The main geological edges are fault lines and the borders of geological or rock bodies of different density, magnetic nature, and so on.

There are many filters are employed to detect and enhance the edges. The horizontal derivatives and vertical derivative are often used to enhance the edge feature. The zero values of vertical derivative have been used to delineate edges in gravity and magnetic

Yongming Yao

ymyao@jlu.edu.cn

1 College of Geoexploration Science and Technology, Jilin University, Changchun 130026, China

2 School of Mechanical Science and Engineering, Jilin University, Changchun 130022, China 
field data (Evjen 1936). Total horizontal derivative (THD) (Cordell 1979; Cordell and Grauch 1985) is a widely used edge detection filer. Nabighian $(1972,1974,1984)$ and Roest et al. (1992) proved that the maxima of the amplitude of the analytic signal (AS) can directly delineate the edges of sources. Hsu et al. (1996) used the higher order derivatives to enhance the edges, called it as the enhanced analytic signal. He pointed out that higher order analytic signal can effectively reduce the interference among adjacent geological bodies and can outline the edges precisely and clearly, but increase the effect of noise. These methods have some disadvantages that they cannot display the strong and weak amplitude anomaly edges simultaneously.

In order to make the strong and weak amplitude edges visible simultaneously, Miller and Singh (1994) used the ratio of vertical derivative to total horizontal derivative to delineate the edges, called it Tilt angle. It was the first published balanced filter, and can balance the amplitude of large and small anomalies effectively. Verduzco and Fairhead (2004) used the total horizontal derivative of Tilt angle (THDR) to outline the edges. Wijns et al. (2005) proposed the Theta map filter to detect the edges. It used analytic signal to normalize the total horizontal derivative. Cooper and Cowan (2006) used the TDX to delineate the edges, which use the vertical derivative to normalize the horizontal gradient amplitude. Ferreira et al. (2013) defined TAHG filter, which is referred to as the tilt angle of the horizontal gradient amplitude. Cooper and Cowan (2008) used the balanced windowed standard deviation to enhance the edges. Ma and Li (2012) proposed the normalized total horizontal derivative (NTHD) to balance the different amplitude edges. This method can effectively balance the edges, but smear out by the nearby points. The identified edges are divergence and have lower resolution. Cooper (2009) used the Hilbert transform to normalize the analytic signal, which can balance the amplitude. But it needs a parameter to control the effectiveness of the filter. Therefore, in order to improve the resolution of the edges, we have to propose some other methods.

Analytic signal is a well-known method. Some previous works have made use of enhancements of the analytic signal (Ma and Du 2012; Cooper 2014, 2015). They are all used to estimate the depth of the magnetic data. In this paper, we use different orders vertical derivatives of potential field data to normalize different orders enhanced analytic signals. We call them as normalized enhanced analytic signals, which can be used to detect the edges.

\section{Normalized enhance analytic signal}

Hsu et al. (1996) defined enhanced analytic signal to reduce the effect of the interference between closely spaced edges and anomalies, which can improve the resolution of the edges effectively. However, they cannot balance the amplitude of edges of different amplitude anomalies. So we can make some improvement to the enhanced analytic signal to solve this problem. With the order of derivative increasing, however, the noise will be magnified. So, we only use the first three order enhanced analytic signals.

The expression of first-order standard analytic signal amplitude is

$$
\mathrm{AS}=\sqrt{\left(\frac{\partial f}{\partial x}\right)^{2}+\left(\frac{\partial f}{\partial y}\right)^{2}+\left(\frac{\partial f}{\partial z}\right)^{2}}
$$

where $f$ is the original gravity and magnetic anomaly data. The expression of second-order enhanced analytic signal is 


$$
\mathrm{SAS}=\sqrt{\left(\frac{\partial f_{z}}{\partial x}\right)^{2}+\left(\frac{\partial f_{z}}{\partial y}\right)^{2}+\left(\frac{\partial f_{z}}{\partial z}\right)^{2}}
$$

where $f_{z}$ is the first order vertical derivative of the potential field. The expression of thirdorder enhanced signal is

$$
\mathrm{TAS}=\sqrt{\left(\frac{\partial f_{z z}}{\partial x}\right)^{2}+\left(\frac{\partial f_{z z}}{\partial y}\right)^{2}+\left(\frac{\partial f_{z z}}{\partial z}\right)^{2}},
$$

where $f_{z z}$ represents the second order derivative of potential field data.

The normalized enhanced analytic signal use different orders vertical derivatives of the potential field data to normalize different orders enhanced analytic signals. These methods avoid the influence of the nearby points and balance the edges of large and small amplitude anomalies simultaneously.

Cooper and Cowan (2006) uses the first order vertical derivative to normalize the total horizontal gradient. Here, we replaced the total horizontal gradient with the analytic signal amplitude to define a new edge detector NAS. The expression of NAS is

$$
\mathrm{NAS}=\tan ^{-1}\left(\frac{\mathrm{AS}}{|\partial f / \partial z|+p \cdot \max (A S)}\right),
$$

where $p$ is a nonnegative constant value decided by the interpreter. In general, the value of $p$ is between 0 and 0.5 . The introduction of $p$ is to avoid producing additional false edges, when anomalies contain both positive and negative anomalies simultaneously. If the anomalies are all positive or all negative, we can set the value of $p$ is zero. If not, the value of $p$ is a positive constant. The large value of $p$ will reduce the effectiveness of balance ability, while the small value of $p$ will enhance the edges of weak amplitude anomaly. The maxima values of NAS locate the edges.

We use second order vertical derivative of potential field data to normalize SAS, the expression is

$$
\mathrm{NSAS}=\tan ^{-1}\left(\frac{\mathrm{SAS}}{\left|\partial^{2} f / \partial z^{2}\right|+p \cdot \max (S A S)}\right) .
$$

The maxima values of NSAS delineate the edges. Here, the parameter $p$ has same property with the $p$ in Eqs. (4) and (5). The NSAS needs the computation of the second order vertical derivative of $f$. We use the following Laplace equation (Blakely 1995) to compute the second vertical derivative:

$$
\frac{\partial^{2} f}{\partial z^{2}}=-\left(\frac{\partial^{2} f}{\partial x^{2}}+\frac{\partial^{2} f}{\partial y^{2}}\right) .
$$

Also, we use third order vertical derivative of potential field data to normalize TAS, the expression is

$$
\text { NTAS }=\tan ^{-1}\left(\frac{\mathrm{TAS}}{\left|\partial^{3} f / \partial z^{3}\right|}\right) .
$$

The maxima values of NTAS delineate the edges. Also, we use the following Laplace equation (Blakely 1995) to compute the third order vertical derivative: 


$$
\frac{\partial^{3} f}{\partial z^{3}}=\frac{\partial^{2} f_{z}}{\partial z^{2}}=-\left(\frac{\partial^{2} f_{z}}{\partial x^{2}}+\frac{\partial^{2} f_{z}}{\partial y^{2}}\right)
$$

In next section, we can get that edge detector NTAS can outline the edges precisely, but there are higher values surrounding the edges, which cause false edges. So, we need modified the NTAS to avoid the effective of the surrounded higher values. The modified NTAS is

$$
\text { MNTAS }=\tan ^{-1}\left(\frac{\operatorname{TAS} \times \frac{\min \left(\partial^{2} f / \partial z^{2}\right)}{\max \left(\partial^{3} f / \partial z^{3}\right)}}{\left|\partial^{2} f / \partial z^{2}\right|+p \cdot \max \left(\operatorname{TAS} \times \frac{\min \left(\partial^{2} f / \partial z^{2}\right)}{\max \left(\partial^{3} f / \partial z^{3}\right)}\right)}\right),
$$

where min and max represent the minimum and maximum value, respectively. They are the statistics results of data in the exploration area. The parameter $p$ has same property with the $p$ in Eq. (8). The maxima values of MNTAS delineate the edges.

In order to demonstrate the feasibility of NAS, NSAS, MNTAS, we choose two other well-known methods to compare the results. They are TDX (Cooper and Cowan 2006) and Tilt angle of the horizontal gradient (TAHG) (Ferreira et al. 2013).

\section{Synthetic model experiments}

To illustrate the benefit of the new methods, we use a series of edge profile of gravity anomaly. Figure 1a shows the vertical plan view of the two identical prisms at a depth to top of 10 and $20 \mathrm{~m}$. Figure $1 \mathrm{~b}$ displays the gravity anomaly of the model in Fig. 1a.
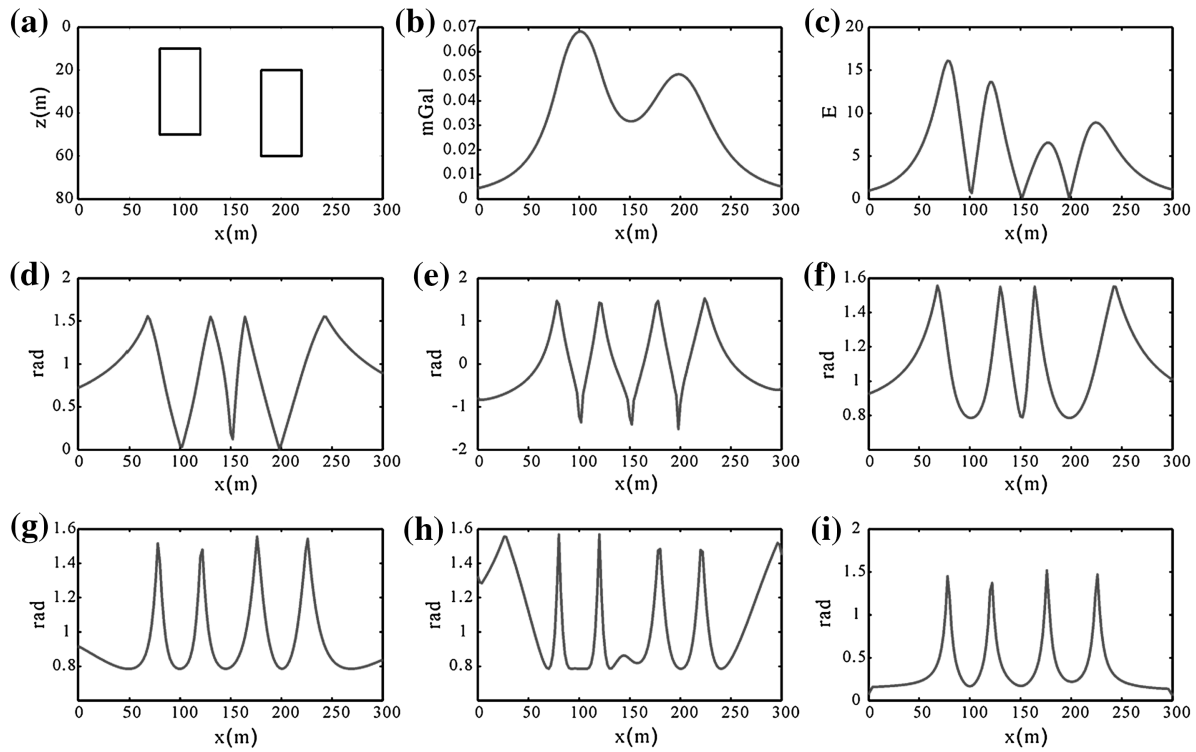

Fig. 1 Profiles showing different edge results from original gravity data. a Vertical plan view of model. b Gravity anomaly of the model in (a). c THD of the data in (b). d TDX of the data in (b). e TAHG of the data in (b). $\mathbf{f}$ NAS of the data in (b), with $\mathrm{p}=0$. $\mathbf{g}$ NSAS of the data in (b), with $\mathrm{p}=0$. $\mathbf{h}$ NTAS of the data in (b). $\mathbf{i}$ MNTAS of the data in (b), with $\mathrm{p}=0$ 
Figure 1c-i display the edges outlined by different methods THD, TDX, TAHG, NAS, NSAS, NTAS and MNTAS. The unbalanced detector THD cannot equalize the edge signal amplitude of shallow and deep sources. By comparing the edge results of the balanced detectors, TAHG, NSAS, NTAS and MNTAS can delineate the edges more precisely than TDX and NAS. Because they are defined by the second order derivative or third order derivative of the potential-field data. However, the TDX and NAS detectors are defined by only using the first order derivatives of potential-field. We can see that the resolution of NAS (Fig. 1f) is slightly better than that of the TDX. The resolutions of NSAS, NTAS and MNTAS are better than TAHG. Although NTAS can extract the edges, it brings some false
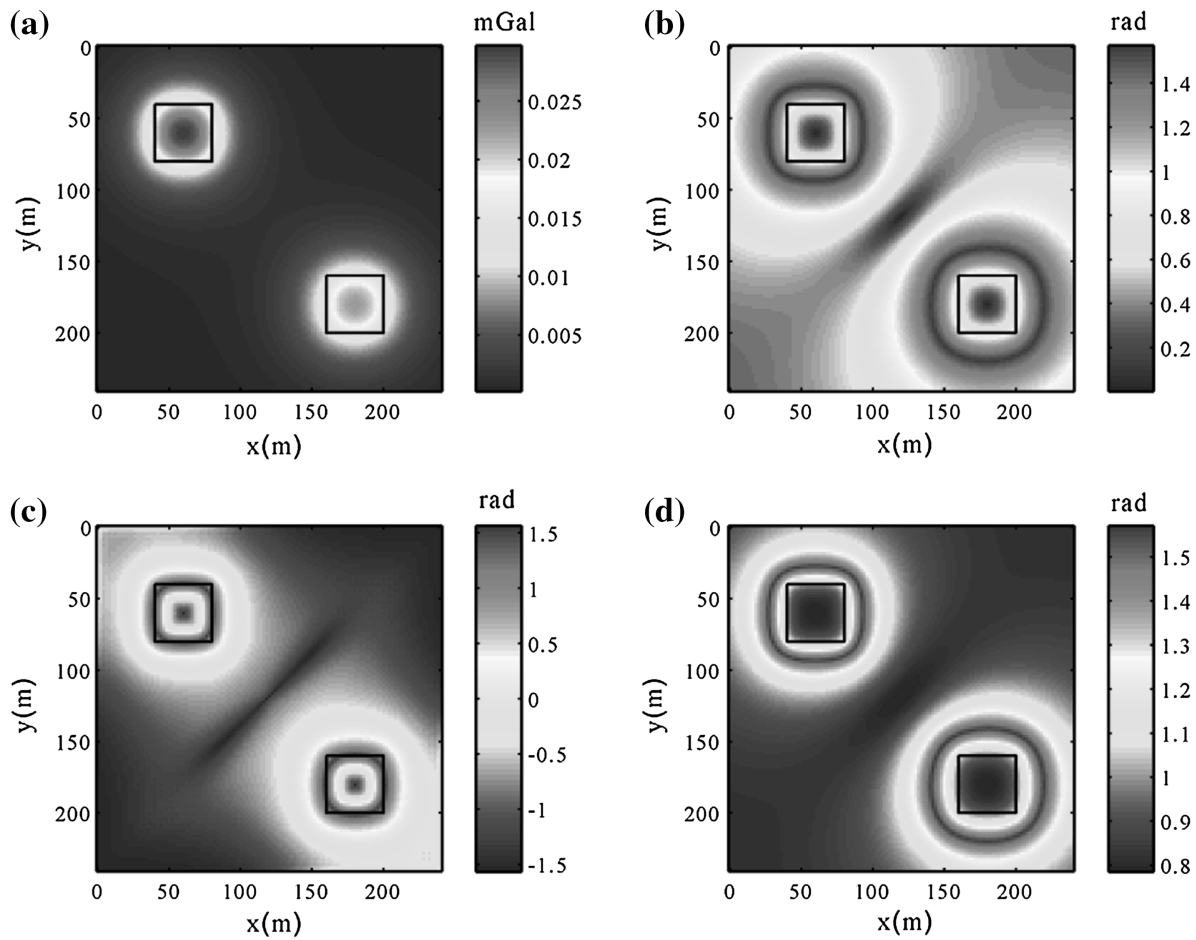

rad

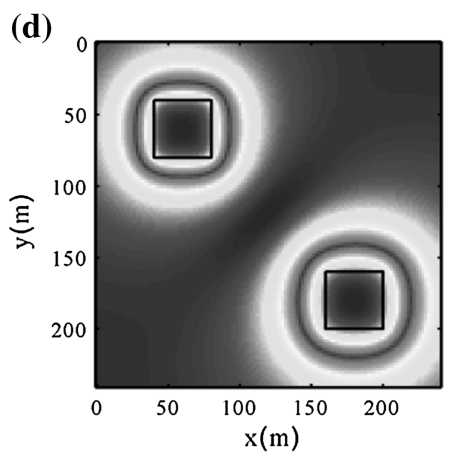

$\mathrm{rad}$
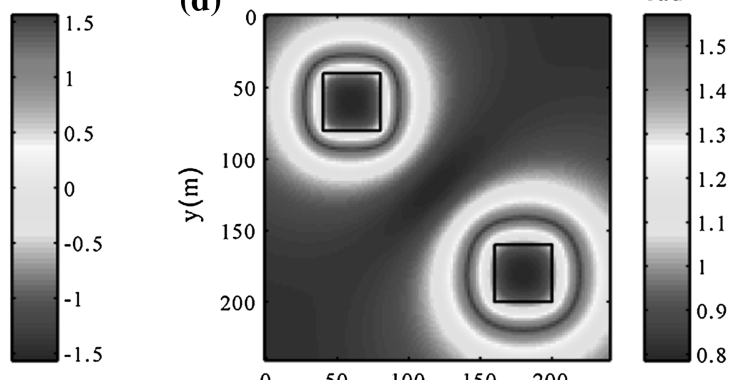

(e)

$\mathrm{rad}$
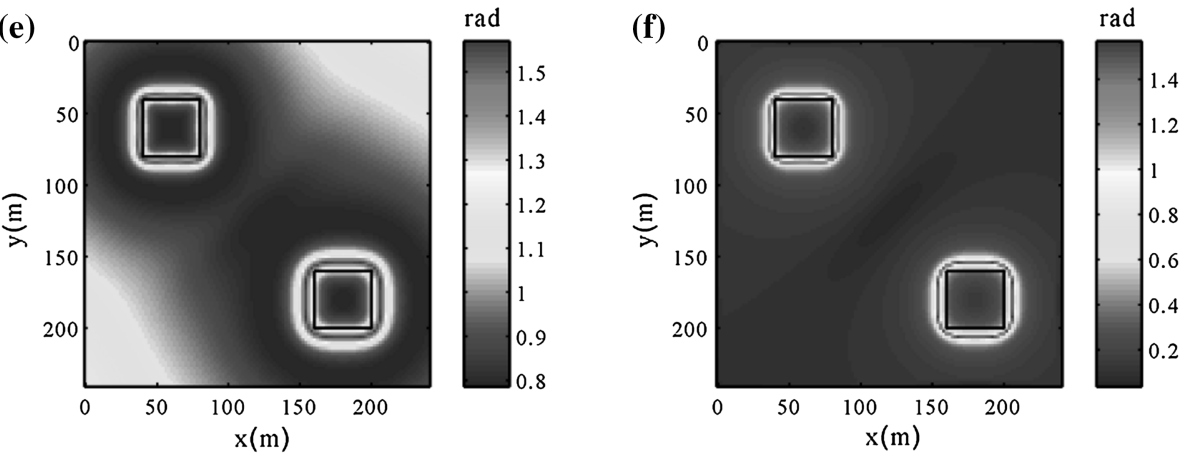

Fig. 2 Edges identified by different methods. a Synthetic gravity anomaly. b TDX of the data in (a). c TAHG of the data in (a). d NAS of the data in (b). e NSAS of the data in (a). $\mathbf{f}$ MNTAS of the data in (a) 
edges surrounding the real edges. The modified version MNTAS can avoid this disadvantages effectively.

We construct another gravity anomaly model which contains two identical prisms with top depths of 10 and $15 \mathrm{~m}$. The dimension of the prism is $40 \mathrm{~m} \times 40 \mathrm{~m} \times 200 \mathrm{~m}$. The residual density is $1 \mathrm{~g} / \mathrm{cm}^{3}$. The size of the exploration area is $240 \mathrm{~m} \times 240 \mathrm{~m}$. The sample grid is $2 \mathrm{~m} \times 2 \mathrm{~m}$. Figure $2 \mathrm{a}$ shows the synthetic gravity anomaly. Figure $2 \mathrm{~b}-\mathrm{f}$ shows the edge results of TDX, TAHG, NAS, NSAS and MNTAS. The edges detected by TDX and NAS are not precise. The TAHG, NSAS and MNTAS can locate the edges

(a)

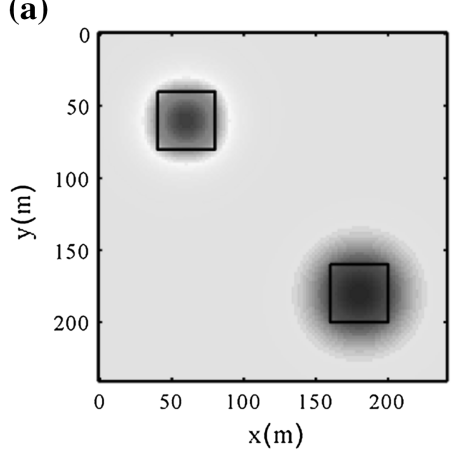

(c)

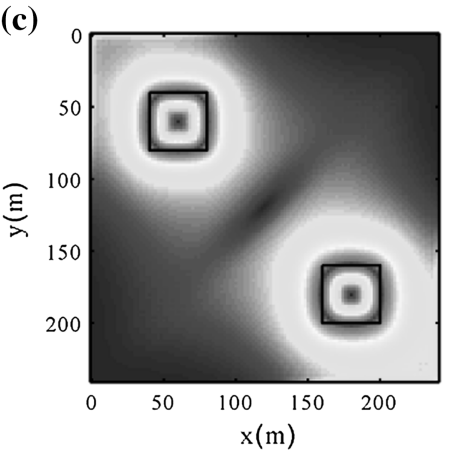

(e)

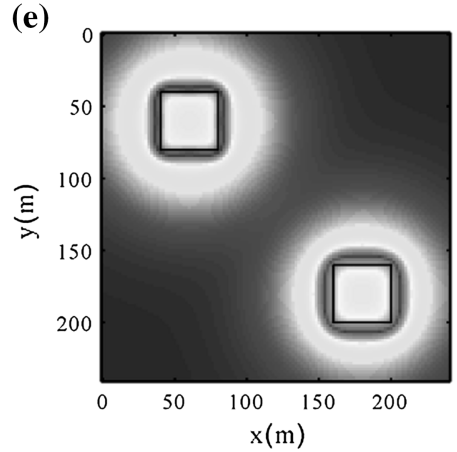

mGal (b)

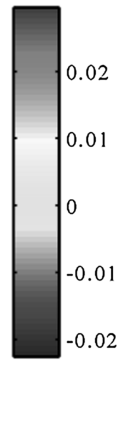

$\mathrm{rad}$

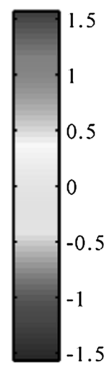

(d)

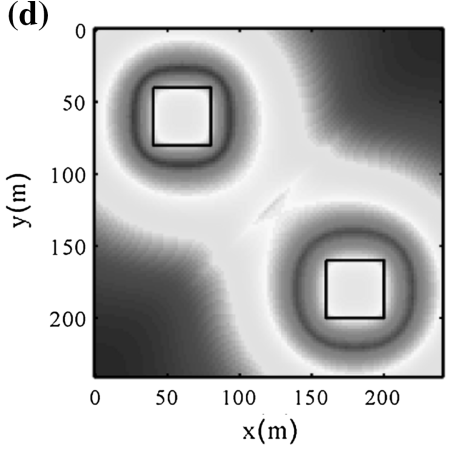

$\mathrm{rad}$

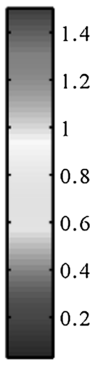

rad $\quad(\mathbf{f})$

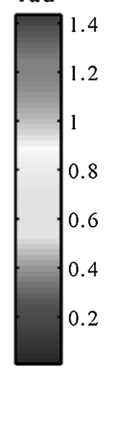

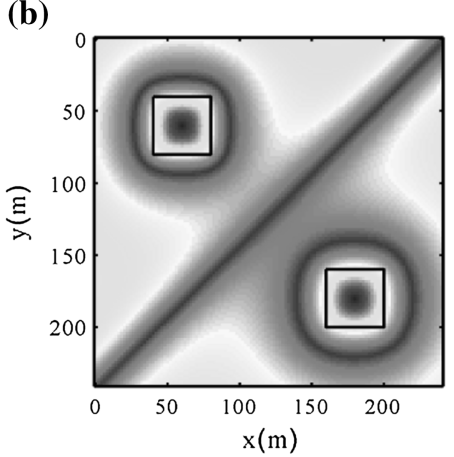

$\mathrm{rad}$

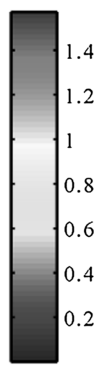


precisely, and the resolution of NSAS and MNTAS is higher than TAHG. Also, Fig. 2e and $\mathrm{f}$ are cleaner than Fig. 2c. However, the TAHG can locate the edges more precisely than NSAS and MNTAS methods. According to the model analysis, the normalized enhanced analytic signal cannot only balance the large and small amplitude edges, but also can get more precise and clear edges compared to some known filters, especially the NSAS and MNTAS.

(a)

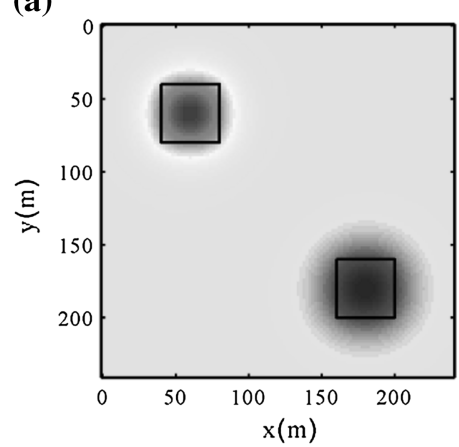

(c)

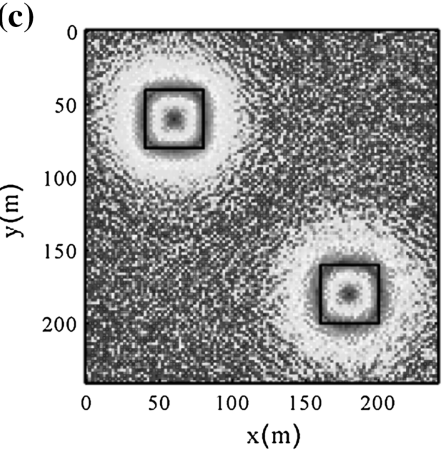

(e)

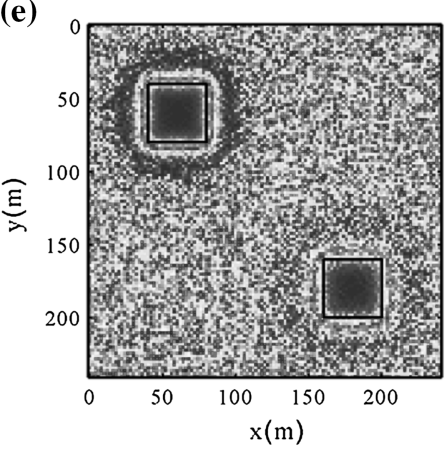

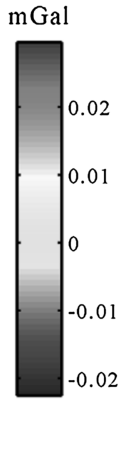

$\mathrm{rad}$

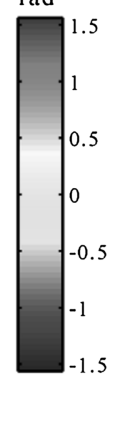

$\mathrm{rad}$

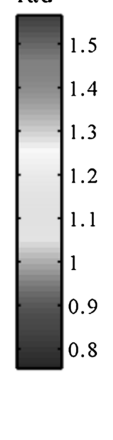

(b)
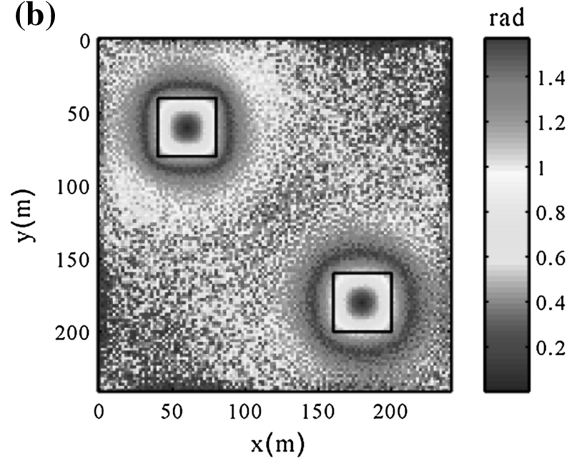

(d)

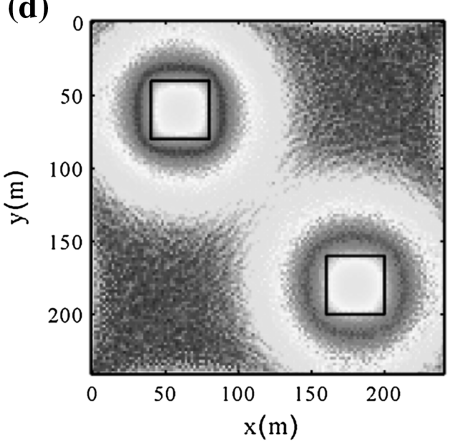

$\mathrm{rad}$

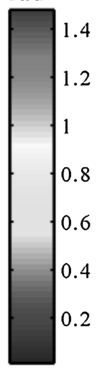

(f)

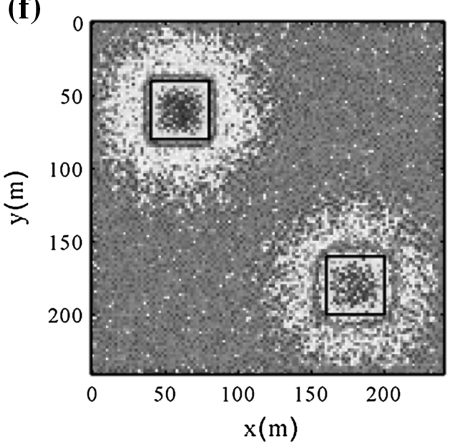

rad

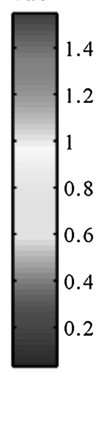

Fig. 4 Edges identified by different methods. a Synthetic gravity anomaly in Fig. 3a contaminated with $0.1 \%$ Gaussian noise. $\mathbf{b}$ TDX of the data in Fig. 2a. c TAHG of the data in Fig. 2a. d NAS of the data in Fig. 2a, with $\mathrm{p}=0.05$. e NSAS of the data in Fig. 2a, with $\mathrm{p}=0.001$. $\mathbf{f}$ MNTAS of the data in Fig. 2a, with $\mathrm{p}=0.01$ 
In order to test the ability to detect the edges of both positive and negative anomalies simultaneously, we construct a model same with the model above, but with the contrasted density of one prism is $1 \mathrm{~g} / \mathrm{cm}^{3}$, the other is $-1 \mathrm{~g} / \mathrm{cm}^{3}$. The synthetic gravity anomaly shows in Fig. 3a. Figure 3b-f display the edges detected by the methods proposed above. We can see that TDX cannot well extract the edges, and it brings the false edge information when the model contains both positive and negative anomalies simultaneously. However, our new can avoid bring any false information.

(a)

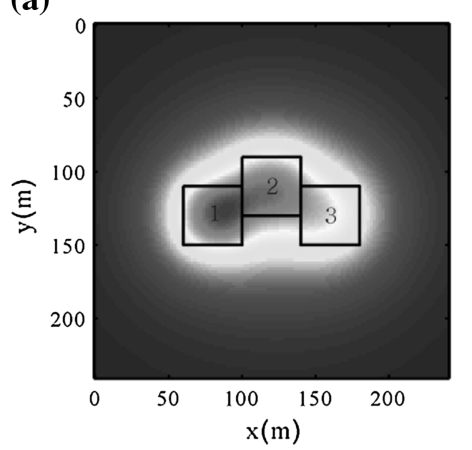

(c)

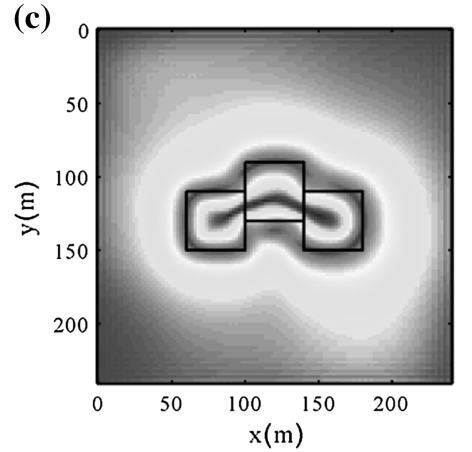

(e)

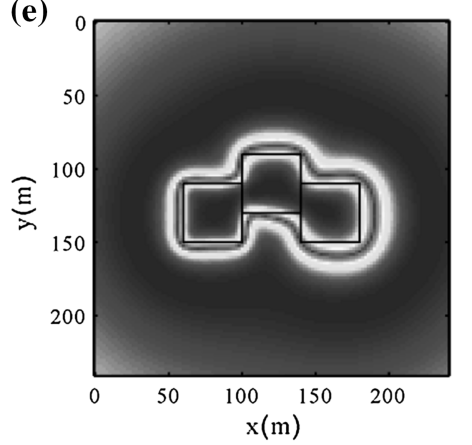

$\mathrm{mGal}$

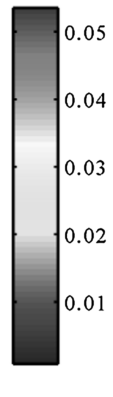

$\mathrm{rad}$

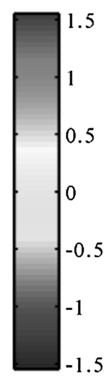

$\mathrm{rad}$

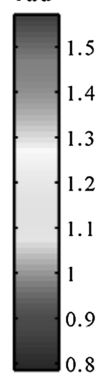

(b)

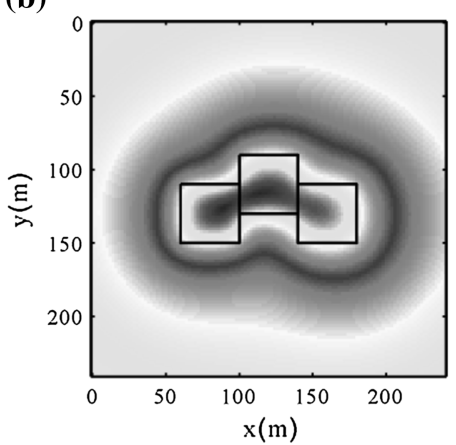

$\mathrm{rad}$

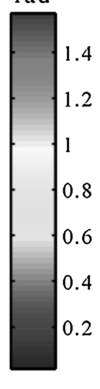

(d)

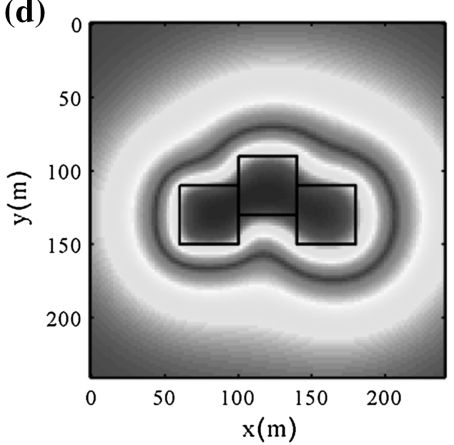

$\mathrm{rad}$

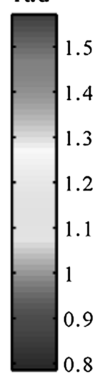

(f)

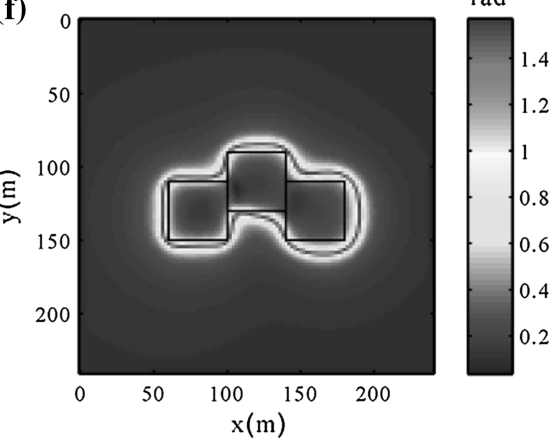

Fig. 5 Edges identified by different methods. a Synthetic gravity anomaly. b TDX of the data in (a). c TAHG of the data in (a). d NAS of the data in (a). e NSAS of the data in (a). f MNTAS of the data in (a) 
The methods proposed by this paper use different order vertical derivatives to normalize the different order enhanced analytic signals. As is known to all, the stability relative to noise will get worse and worse, when the derivative order increasing. Therefore, in order to test the stability of the methods, we add $0.1 \%$ Gaussian noise to the data in Fig. 3a. Figure $4 \mathrm{a}$ shows the noisy data. The edge results detected by different means are shown in Fig. 4b-f. We can see that TAHG, NSAS and MNTAS perform poor, but the TAHG performance is better than NSAS and MNTAS techniques. Because they are defined by second order derivative and third order derivative, which increase the noise influence. Therefore, we need to remove the noise first before we use our methods to detect the edges.

In order to illustrate the resolution of the new method to detect the edges of multi closely geological bodies, we construct a gravity anomaly model produced by three closely prismatic sources. Figure 5a shows the gravity anomaly of three prisms with the top depths of 10,15 and $20 \mathrm{~m}$. The dimensions of the prisms are $40 \mathrm{~m} \times 40 \mathrm{~m} \times 200 \mathrm{~m}$. The residual density is $1 \mathrm{~g} / \mathrm{cm}^{3}$. The size of the exploration area is $240 \mathrm{~m} \times 240 \mathrm{~m}$. The sample grid is $2 \mathrm{~m} \times 2 \mathrm{~m}$. Fig. 5b-f shows the TDX, TAHG, NAS, NSAS and MNTAS of the data in Fig. 5a. By comparing the results, we can see that NSAS and MNTAS can not only delineate the edges of the sources clearly and precisely, but also give better resolution of the edges than any of other filters.

(a)

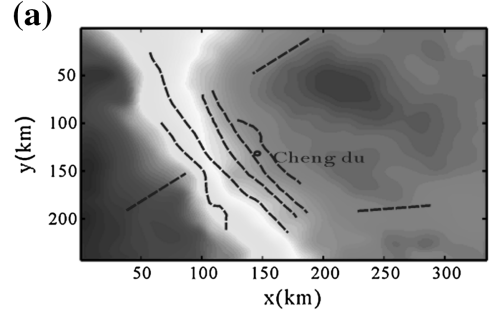

(c)

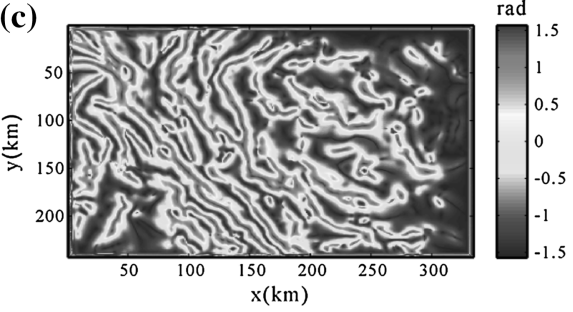

(e)

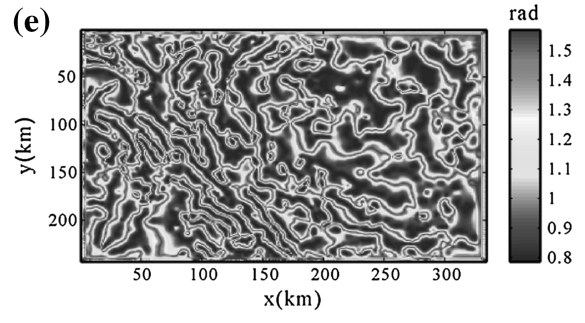

(b)

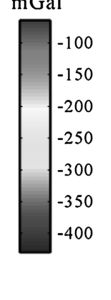

$\prod^{\mathrm{rad}} 1.5 \quad(\mathbf{d})$

(d)
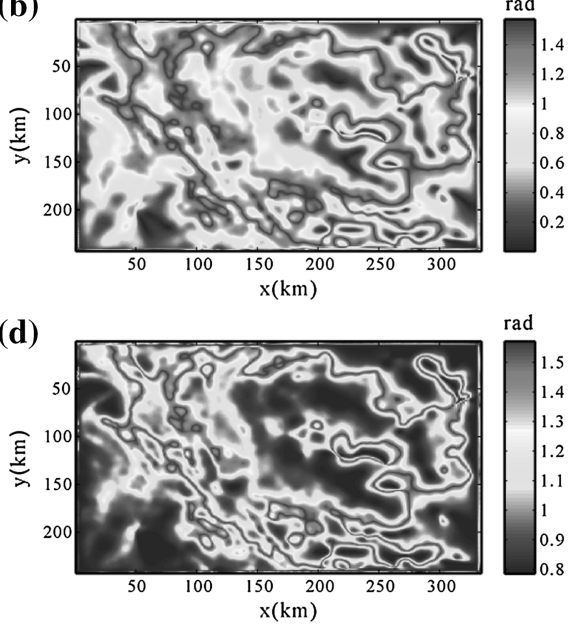

(f)

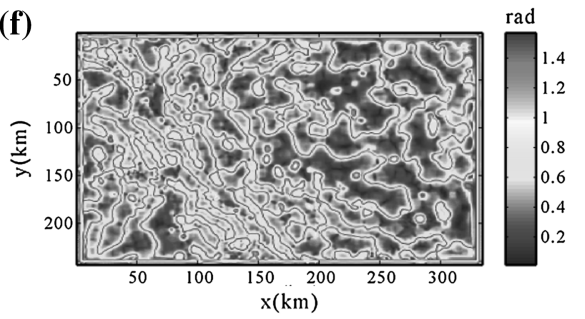

Fig. 6 Edges identified by different methods. a Gravity anomalies in Sichuan basin, China. b TDX of the data in (a). c TAHG of the data in (a). d NAS of the data in (a), with $p=0.0001$. e NSAS of the data in (a), with $\mathrm{p}=0.0001$. $\mathbf{f}$ MNTAS of the data in (a), with $\mathrm{p}=0.0001$ 
Li (2006) has pointed that the analytic signal and enhanced analytic signal are dependent on the earth's magnetic field and remanent magnetization. The horizontal derivatives of the magnetic anomaly are sensitive to magnetization direction ( $\mathrm{Ma}$ and Li 2012). So we need to reduce the magnetic anomaly to the pole before using the normalized enhanced analytic signal to enhance the edges of the magnetic data.

\section{Application to real gravity and magnetic data}

In order to test the application in real cases, we use the methods to the process the gravity data from Sichuan basin, Southwest, China and the magnetic data from Zhurihe, Northeast, China. The first order vertical derivative of the real data is computed by a directional derivative algorithm in the frequency domain. The second and third orders vertical derivatives are computed by Eqs. (6) and (8), respectively.

Figure 6a shows the gravity data from Sichuan basin, Southwest, China, which collected from the bouguer gravity anomaly, on a scale of 1:1,000,000, provided by the National Administration of Surveying, Mapping and Geoinformation, China. We interpolate the data with a grid interval $500 \mathrm{~m}$. The black dotted lines in Fig. 6a mark the known fracture in a horizontal position. Figure $6 \mathrm{~b}-\mathrm{f}$ shows the TDX, TAHG, NAS, NSAS

(a)

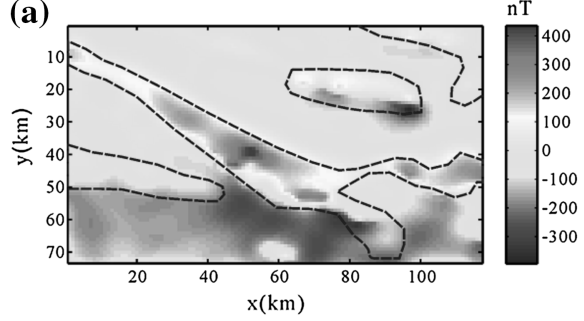

(c)

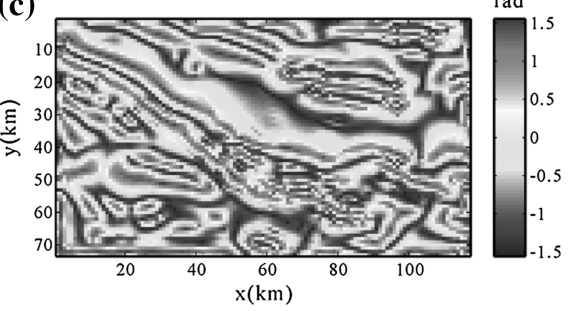

(e)

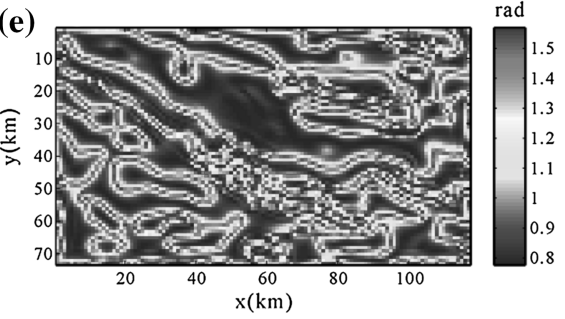

(b)

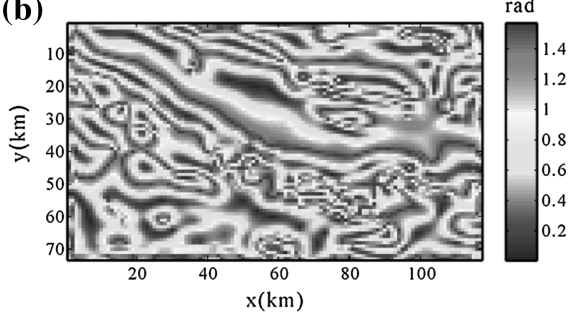

(d)

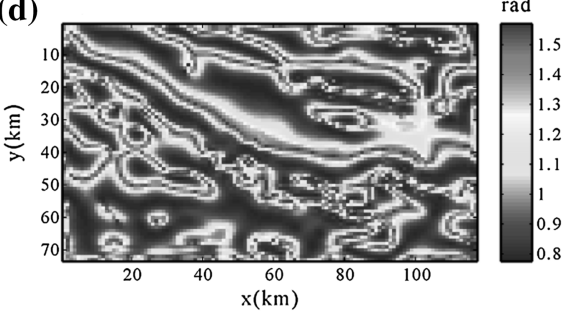

(f)

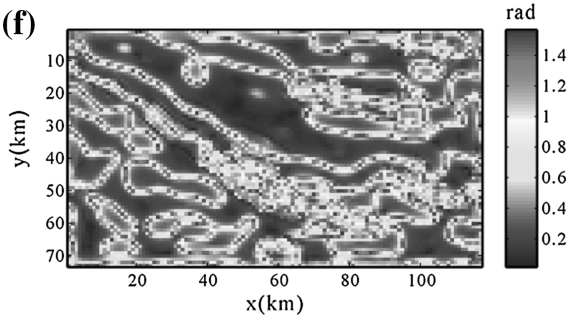

Fig. 7 Edges identified by different methods. a Reduce to pole of magnetic anomalies in Zhurihe, China. b TDX of the data in (a). c TAHG of the data in (a). d NAS of the data in (a), with p $=0.0001$. e NSAS of the data in (a), with $\mathrm{p}=0.0001$. $\mathbf{f}$ MNTAS of the data in (a), with $\mathrm{p}=0.0001$ 
and MNTAS of the data in Fig. 6a. We can get that TDX and NAS get the similarity edge results. But NAS is slight better than TDX. TAHG, NSAS and MNTAS can identify more edge information than TDX and NAS. Although our new methods and TDX may introduce some ellipse-shaped artifacts in identified edges, the resolution of NSAS and MNTAS is higher than TAHG.

Figure 7a shows the reduction to pole of magnetic data from Zhurihe, Northeast, China. The sampling interval of the data is $20 \mathrm{~m}$ and the size of study area is $73 \times 117 \mathrm{~km}$, and the flight height is $1500 \mathrm{~m}$, and the line spacing is $500 \mathrm{~m}$. The Neoproterozoic supersequence consists predominantly of continental sediments and has decreased the intensity of magnetic signatures, except for some iron-rich sandstone dykes. The dataset is dominated by the near linear anomalies generated by the nearly SE-NW trending dykes and the iron structures dominate the map. The black dotted lines in Fig. 7a mark the known fracture in a horizontal position. Figure 7b-f shows the TDX, TAHG, NAS, NSAS and MNTAS of the data in Fig. 7a. NSAS and MNTAS can extract the edges more clearly. They have obvious advantages in enhancing the edges of the low amplitude sources, making an improved geological interpretation possible.

\section{Conclusion}

In this paper, we present three new edge detectors NAS, NSAS and MNTAS. They all apply the different orders vertical derivatives of the potential field data to normalize the different orders enhanced analytic signals. The introduction of higher order derivatives enhanced the sensitivity of NSAS and MNTAS to the noise. Therefore, we must filter the data firstly in real cases. They can not only balance the large and small amplitude edges, but also give a higher resolution, and can delineate the edges more clearly and precisely. The detectors have been demonstrated on synthetic and measured gravity and magnetic data. They show that NSAS and MNTAS can get a higher resolution edges, and can bring out more details, making an improved geologic interpretation possible.

Acknowledgments The authors are grateful to the National High Technology Research and Development 863 Program of China (Grant No. 2013AA063930) for supporting this work.

\section{References}

Blakely RJ (1995) Potential theroy in gravity and magnetic applications. Cambridge University Press, Cambridge

Cooper G (2009) Balancing images of potential-field data. Geophysics 74:L17-L20

Cooper GRJ (2014) The automatic determination of the location and depth of contacts and dykes from aeromagnetic data. Pure Appl Geophys 171:2417-2423

Cooper GRJ (2015) Using the analytic signal amplitude to determine the location and depth of thin dikes from magnetic data. Geophysics 80(1):J1-J6

Cooper G, Cowan D (2006) Enhancing potential field data using filters based on the local phase. Comput Geosci 32:1585-1591

Cooper G, Cowan D (2008) Edge enhancement of potential-field data using normalized statistics. Geophysics 73:H1-H4

Cordell L (1979) Gravimetric expression of graben faulting in Santa Fe Country and the Espanola Basin. 30th Field conference New Mexico. New Mexico Geological Society Guidebook, 59-64

Cordell L, Grauch VJS (1985) Mapping basement magnetization zones from aeromagnetic data in the San Juan basin, New Mexico. In: Hinze WJ (ed) The utility of regional gravity and magnetic anomaly society of exploration geophysics, p 181-197 
Evjen HM (1936) The place of the vertical gradient in gravitational interpretations. Geophysics 1:127-136

Ferreira FJF, Douza J, Bongiolo ABS, Castro LG (2013) Enhancement of the total horizontal gradient of magnetic anomalies using the tilt angle. Geophysics 78(3):J33-J41

Hsu SH, Sibuet JC, Shyu CT (1996) High-resolution detection of geologic boundaries from potential-field anomalies: an enhanced analytic signal technique. Geophysics 61:373-386

Li X (2006) Understanding 3D analytic signal amplitude. Geophysics 71(2):L13-L16

Ma G, Du X (2012) An improved analytic signal technique for the depth and structural index from 2D magnetic anomaly data. Pure Appl Geophys 169:2193-2200

Ma G, Li L (2012) Edge detection in potential fields with the normalized total horizontal derivative. Comput Geosci 41:83-87

Miller HG, Singh V (1994) Potential field tilt-a new concept for location of potential field sources. J Appl Geophys 32:213-217

Nabighian MN (1972) The analytic signal of two-dimensional magnetic bodies with polygonal crosssection: its properties and use for automated anomaly interpretation. Geophysics 37:507-517

Nabighian MN (1974) Additional comments on the analytic signal of two-dimensional magnetic bodies with polygonal cross-section. Geophysics 39:85-92

Nabighian MN (1984) Toward a three-dimensional automatic interpretation of potential field data via generalized Hilbert transforms: fundamental relations. Geophysics 49:780-786

Roest WR, Verhoef J, Pilkington M (1992) Magnetic interpretation using the 3-D analytic signal. Geophysics 57:116-125

Verduzco B, Fairhead JD (2004) New insights into magnetic derivatives for structural mapping. Lead Edge 23:116-119

Wijns C, Perez C, Kowalczyk P (2005) Theta map: edge detection in magnetic data. Geophysics 70:L39L43 